\title{
Desempenho Zootécnico e Tabela de Alimentação de Girinos de Rã-Touro(Rana catesbeiana) Criados no Sistema Anfigranja ${ }^{1}$
}

\author{
Samuel Lopes Lima ${ }^{2}$, Alex Poeta Casali ${ }^{3}$, Cláudio Angelo Agostinho ${ }^{4}$
}

\begin{abstract}
RESUMO - Objetivou-se com a execução deste trabalho avaliar, em nível de campo, o desempenho zootécnico de girinos de rã-touro (Rana catesbeiana) criados em ranários comerciais que utilizam o sistema anfigranja, e simultaneamente estimar os valores de consumo diário de ração para compor uma tabela de referência para a alimentação dos animais. Os resultados encontrados nas unidades de observações monitoradas (aproximadamente 249 mil animais) atingiram os seguintes índices médios: a mortalidade (em \%) variou de: 0,7 a 35,2 (média 5,9\%); o ganho de peso (g. p/dia): de 0,03 a 0,22 (média 0,11 g/dia); e a conversão alimentar: de 0,92 a 2,75: 1; (média 1,50 : 1). Comparados com dados preliminares, tais resultados indicam uma significativa otimização dos índices zootécnicos, demonstrando que houve um relativo ganho de produtividade com o aperfeiçoamento das técnicas de manejo implementadas. A redução do valor médio da conversão alimentar aparente foi provocada principalmente pelo adequado uso da tabela de referência, cujos valores foram ratificados durante o trabalho de monitoramento das unidades. Tal tabela possibilita o cálculo da quantidade de alimento a ser oferecido diariamente, baseado nos peso médio dos girinos e na estimativa do percentual de consumo, para temperatura da água entre 18 a 29 graus centígrados, (girinos de 0,1 a 26 gramas de peso médio).
\end{abstract}

Palavras-chave: criação de girinos; índices zootécnicos; rã-touro; ranicultura; Sistema Anfigranja; tabela de alimentação.

\section{Performance of Bullfrog Tadpoles (Rana catesbeiana) Raised in the "Amphifarm" System and Feeding Tables}

\begin{abstract}
The objective of this work was to evaluate (under field conditions), the performance of bullfrog tadpoles (Rana catesbeiana) raised on commercial frog farms using the "Amphifarm" Systems, and, at the same time, to estimate daily food intake, in order to obtain a reference table for feeding the animals. The results found on the observation units (approximately 249 mil animals) showed the following indexes: mortality $(\%)$ : range from 0.7 to 35.2 , the average being 5.9 ; weight gain (g/day) varied from 0.03 to 0.22 , the average being 0.11 ; feed conversion ranged from 0.92 to $2.75: 1$, the average being 1.50:1. Compared to previous data, these results indicate a significant improvement in performance indexes, showing that there was a relative gain in productivity due to the improvement of management techniques. The reduction in average feed conversion was caused mostly by adequate use of the reference table, the values of which were confirmed during a follow-up of the observation units. This table allows the calculation of daily food offer, based on tadpole average weight and on estimated intake as a percentage of body weight, for water temperatures varying from 18 to $29 \mathrm{C}$ and body weight ranging from 0.1 to $26 \mathrm{~g}$.
\end{abstract}

Key Words: tadpole rearing; performance indexes; bullfrog; frog production; "Amphifarm” system; feeding table.

\section{Introdução}

A criação de rãs é uma atividade em fase emergente e em franco crescimento no Brasil, com mais de 600 ranários e 15 indústrias de abate e beneficiamento, implantados principalmente na Região Sudeste do país, segundo levantamento realizado por Lima et al. (1999). Os ranários foram construídos com várias alternativas, ou propostas de instalações e técnicas de manejo, dentre elas o Sistema Anfigranja. No levantamento também foram constatadas as dificuldades dos ranicultores em absorver tecnologia, particularmente quanto às técnicas de manejo da criação de girinos.

As características das instalações do sistema anfigranja de criação intensiva de rãs foram definidas a partir do desenvolvimento de vários protótipos, aliados às técnicas de manejo sistematizadas para cada um dos setores da criação: reprodução, girinos e recria. Tal sistema foi proposto por Lima \& Agostinho $(1988,1992)$ e encontra-se ainda em fase de consolidação tecnológica, apesar de estar em domínio público por parte de alguns ranicultores. Nele, o setor de girinos é composto de instalações (tanques seme-

\footnotetext{
${ }_{1}^{1}$ Pesquisa parcialmente financiada pelo CNPq (bolsas aos pesquisadores).

2 Pesquisador visitante (bolsista do CNPq). E.mail: samuel@ufv.br

3 Zootecnista (bolsista do CNPq). E.mail: apcasali@lince.tdnet.com.br

4 Professor FMVZ/UNESP - Botucatu - SP. E.mail: agostinho@fca.unesp.br
} 
lhantes aos da piscicultura) construídas com detalhes especiais para possibilitar o manejo dos animais com técnicas sistematizadas desde a obtenção dos embriões, até o final da metamorfose (Lima \& Agostinho 1988, 1992; Lima, 1997).

Nos últimos anos, alguns detalhes de manejo e aperfeiçoamento nas instalações foram implementados (Lima, 1997), refletindo nos resultados de produtividade dos animais nos ranários que empregam o sistema. As instalações são constituídas de tanques de alvenaria, de formato circular ou retangular, com eficiente sistema de drenagens dos resíduos e circulação da água através de um conjunto hidráulico de alta pressão. Em 1998, Lima \& Valles apresentaram dados preliminares dos índices de produtividade de girinos de rã-touro no sistema anfigranja: mortalidade estimada em 7,65\% (média geral), excluídos os casos de mortandades provocadas por eventuais enfermidades ou erros de manejo; ganho de peso diário 0,01 a 0,22 g (média geral $0,10 \mathrm{~g} / \mathrm{dia}$ ); conversão alimentar aparente (ração oferecida : ganho de peso no período), estimada entre 0,84 a 3,08:1 (média geral 1,69: 1).

Os girinos e as rãs são pecilotérmicos, ou seja, o metabolismo varia de acordo com a temperatura do meio onde vivem (Brattstrom, 1979). Assim como outros organismos aquáticos e pecilotérmicos que são cultivados (peixes, camarões, moluscos), o produtor tem sérias dificuldades de alimentar os animais adequadamente. Como as variações da temperatura afetam o consumo de alimento, o que se observa é que, ora existe sobra de alimento, ora a oferta pode ser insuficiente. $\mathrm{O}$ primeiro fato reflete no comprometimento da qualidade da água e eleva o custo da produção pelo consumo excessivo da ração, principal alimento. No segundo, seu reflexo pode resultar no aumento do tempo de cultivo, promover inanição ou até mesmo canibalismo entre os girinos.

Para orientar no manejo alimentar, Lima \& Agostinho (1988) apresentaram uma tabela de referência para a oferta diária da ração, baseada no percentual do peso médio dos animais (5 a 12\%). Tal tabela apresentava uma série de limitações: foi estabelecida em nível de laboratório, em aquários, portanto os animais não estavam sujeitos às variações das intempéries climáticas (radiação solar, vento, chuva, etc), e além deste fato, estava limitada ao intervalo de 0,1 a 5,0 gramas do peso médio. Portanto na prática, a tabela era de pouca utilidade para o ranicultor, que até então tem oferecido o alimento de forma aleatória.
Estudos similares sobre o consumo alimentar de girinos de rã-touro foram realizados por Culley et al. (1977), que concluíram a necessidade de uma oferta diária de alimento equivalente a 12-13\% de biomassa dos animais alojados em determinado tanque. Ratificando tal informação, Fontanello et al. (1982) ofereceram ração (para coelhos) com 15\% de PB, pulveriza$\mathrm{da}$, a girinos de rã-touro e encontraram maior ganho de peso quando a oferta diária correspondeu a $13 \%$ do peso corporal dos animais. Em 1985, Soares et al., oferecendo concentrado protéico comercial, com $36 \%$ de $\mathrm{PB}$, encontraram maior ganho de peso com a oferta de $18 \%$ do peso vivo.

Avaliação do consumo de ração de girinos de rãtouro alimentados com diferentes proporções de ração em relação ao peso corporal também foi realizada por Mello et al. (1997), em condições de laboratório, com renovação diária de $200 \%$ do volume do aquário, concluindo que, quando foi administrada a proporção diária de $13 \%$ do peso corporal, os animais obtiveram maior ganho de peso, enquanto a conversão alimentar foi mais eficiente para a proporção de 3 a $9 \%$ e de 2 a $7 \%$.

Tendo em vista a necessidade de se promover o desenvolvimento tecnológico do Sistema Anfigranja objetivou-se com a execução deste trabalho a avaliação, em nível de campo, do desempenho zootécnico de girinos da rã-touro, Rana catesbeiana (Shaw, 1882) criados em ranários comerciais que utilizam o referido sistema, e simultaneamente, efetuar a calibração da tabela de referência para o adequado arraçoamento diário dos animais, em função da temperatura média da água.

\section{Material e Métodos}

No período de março de 1997 a fevereiro de 2001, foram monitorados vários ciclos completos de produção de três ranários, localizados respectivamente nos municípios de Anchieta (ES), Coimbra e Visconde do Rio Branco (MG), aqui denominados de Unidades de Observações (respectivamente UO-1; UO-2 e UO-3). A amostra foi constituída da população de todos animais alojados em determinados tanques de criação (denominados de lotes) durante o período de permanência, até concluírem a metamorfose. Constam na Tabela 1 as características físicas de cada unidade e o respectivo número de lotes de animais monitorados. Cada lote de girinos foi avaliado em períodos e ciclos de produção diferentes, de acordo com procedimen- 
Tabela 1 - Características do setor de girinos dos ranários monitorados

Table 1 - Characteristics of the tadpole section in the observed frog farms

\begin{tabular}{|c|c|c|c|c|c|}
\hline $\begin{array}{l}\text { Município } \\
\text { (Estado) } \\
\text { City (State) }\end{array}$ & $\begin{array}{l}\text { Altitude (m) } \\
\text { Altitude (m) }\end{array}$ & $\begin{array}{c}\text { Temperatura } \\
\text { média da água }\left({ }^{\circ} \mathrm{C}\right) \\
\text { Average water } \\
\text { temperature }\left({ }^{\circ} \mathrm{C}\right)\end{array}$ & $\begin{array}{c}\text { Número e Dimensões } \\
\text { dos tanques }(\mathrm{m}) \\
\text { Number and tank } \\
\text { dimensions }(m)\end{array}$ & $\begin{array}{l}\text { Capacidade } \\
\text { de alojamento } \\
\text { Shelter } \\
\text { capacity } \\
\end{array}$ & $\begin{array}{c}\mathrm{N}^{\mathrm{o}} \text { de lotes } \\
\text { monitorados } \\
\text { Number of groups } \\
\text { observed }\end{array}$ \\
\hline UO-1 & 200 & 25 & (12) $3,0 \times 2,0 \times 1,0$ & 36.000 & 59 \\
\hline UO-2 & 650 & 20 & (6) $3,0 \times 2,0 \times 1,0$ & 18.000 & 14 \\
\hline UO-3 & 450 & 24 & (6) $3,0 \times 2,0 \times 1,0$ & 18.000 & 10 \\
\hline
\end{tabular}

tos descriminados adiante. Foram monitorados 83 lotes, em um total aproximado de 249 mil animais.

Os desenhos ou modelos de cada tanque seguiram as características descritas por Lima, (1997). Todos os tanques monitorados foram construídos em alvenaria, com o formato retangular, piso com inclinação central de $12 \%$, confluindo para uma caixa de coleta dos resíduos protegida com tela na parte superior para evitar a fuga dos animais. O esgotamento total ou parcial da água foi realizado por cotovelo roscável, que também tem a função de manter o nível da coluna d'água do tanque. Dotado de um conjunto hidráulico de alimentação de água com tubos de alta pressão, entrada alternativa pela superfície ou pelo fundo, com orifícios na tubulação para propiciar jatos direcionados para a caixa de drenagem, auxiliando na retirada do material sedimentar (restos de ração fezes e outros resíduos). $\mathrm{Na}$ ausência deste conjunto, alternativamente se utilizou o fluxo contínuo de água. Nos dois casos o fluxo de água era paralisado para a oferta do alimento durante 20 a 30 minutos. Diariamente, pela manhã, antes da primeira alimentação e no final da tarde, o cotovelo era movimentado para esgotar os resíduos.

Apesar de os ranicultores terem acesso às informações da tecnologia, cada ranário foi construído com pequenas diferenças construtivas, de acordo com a criatividade de cada um. Portanto, a primeira iniciativa foi a correção dessas diferenças, no sentido de padronizar tais características para minimizar a ação das variáveis. Foram anotadas as temperaturas da água dos tanques, diariamente às $7 \mathrm{~h}$.

A densidade utilizada em todos os tanques foi de dois litros de água para cada animal. A estimativa do número de girinos foi obtida pelo cálculo entre o peso total, ou biomassa, de cada tanque, dividido pelo peso médio de cada indivíduo. Por sua vez, o peso médio era estimado a partir de uma amostra constituída de $10 \%$ do total de girinos alojados.

Para estabelecer os valores da tabela de alimentação, a primeira iniciativa consistiu em ampliar a faixa de peso, de 0,1 a 5,0g (estabelecido em Lima \& Agostinho, 1988), para 0,1 a 26 g. Para a montagem dessa primeira tabela, adotou-se o seguinte procedimento: diariamente, o alimento era pesado, em valor superior ao esperado de ser consumido pelos girinos de cada tanque, colocados em recipientes identificados. O tratador administrava o alimento em parcelas, pulverizado, na superfície. Em cada parcela, a ração era oferecida, gradativamente, até saciar o aparente interesse dos animais pelo alimento. Ao final do dia, o peso do alimento oferecido era conhecido através da simples subtração do peso da ração restante no recipiente. Tal procedimento foi realizado no período de abril a setembro de 1999, época em que a temperatura da água dos tanques oscilou entre 18 e $20^{\circ} \mathrm{C}$. $\mathrm{O}$ alimento era constituído de ração comercial, extrusada, para truta em fase inicial, moída em partícula fina, (peneira malha $2 \mathrm{~mm}$ ). Sua composição apresentava os seguintes níveis de garantia pelo fabricante: proteína bruta (PB), $40 \%$; extrato etéreo (EE) 4\%; fibra bruta (FB) 6\%; minerais 14\%; cálcio (Ca): $3 \%$; fósforo $(\mathrm{P}): 1 \%$; e umidade: $12,5 \%$.

De posse dos valores de peso do alimento oferecido e do peso médio dos animais, foram estimados os respectivos percentuais correspondentes, montando-se assim a tabela para a faixa de 18 a $20^{\circ} \mathrm{C}$ (temperatura do período). Através de simulações quantitativas, obedecendo à regra da proporcionalidade, foram estimados os valores correspondentes para as faixas de intervalos de temperatura: 18 a $20^{\circ} \mathrm{C} ; 21$ a $23^{\circ} \mathrm{C} ; 24$ a $26^{\circ} \mathrm{C}$; 27 a $29^{\circ} \mathrm{C}$. Composta a tabela, os valores estimados, para cada intervalo de temperatura, foram 
ratificados ou aferido por meio de vários monitoramentos realizados no período de março de 1998 a fevereiro de 2001, em um procedimento aqui denominado de calibração.

Para a calibração dos valores da tabela definitiva adotou-se os seguintes passos: semanalmente, retirava-se uma amostra, de cada tanque para se obter o peso médio dos girinos. A tabela indicava o percentual a ser utilizado para o cálculo da quantidade estimada de ração a ser consumida, em função da biomassa existente no tanque (peso total de girinos). $\mathrm{O}$ peso total do alimento a ser oferecido, era dividido em quatro a sete parcelas diárias (dependendo da temperatura da água). Ou seja, o cálculo do alimento a ser oferecido era efetuado de acordo com o peso médio e a temperatura matinal observada durante a semana, obedecendo as faixas ou intervalos acima definidos. Observações sistemáticas do tratador, verificando se a quantidade oferecida estava sendo suficiente para "saciar" a fome dos girinos, eram anotadas para as possíveis correções dos valores estimados, seguindo assim o mesmo procedimento efetuado para a primeira tabela.

Foram procedidas, para cada tanque, em cada período, em todas as unidades observadas, as anotações das mortes ocasionais, do peso médio inicial e final de cada lote de girinos, e do alimento oferecido, para os respectivos cálculos da: taxa de mortalidade, do ganho de peso e da conversão alimentar aparente. Foi considerado como consumido, todo alimento oferecido.

Os mesmos índices zootécnicos foram estimados, por faixa de pesos, na unidade UO-1, durante o período de setembro de 1999 a fevereiro de 2001. Para tal foram monitorados 59 lotes, em ciclos de produção sucessivos, amostrando-se aproximadamente 176 mil girinos. As faixas de peso consideradas foram: a fase inicial da vida dos girinos (até atingir um grama), de um a cinco gramas (crescimento), e acima de cinco gramas, até atingirem o clímax da metamorfose. Tal classificação se justifica pelas diferenças adotadas no manejo de rotina, de acordo com técnica proposta por Lima (1997).

\section{Resultados e Discussão}

Constam na Tabela 2 os valores de taxa de mortalidade, ganho de peso médio diário e a conversão alimentar dos girinos de rã-touronas três unidades monitoradas (UO-1, UO-2; UO-3). Na Tabela 3, apresentam-se dados semelhantes, porém classificados por faixas de peso, de uma das unidades avaliadas (UO-1).

Os resultados da presente avaliação indicaram clara melhoria na produtividade quando comparados com as avaliações preliminares efetuadas por Lima \& Agostinho (1992), Lima et al. (1997) e Lima \& Valles (1998). A diminuição dos valores de conver-

Tabela 2 - Valores mínimos, máximos e médios dos índices zootécnicos de girinos de rã-touro nas três unidades monitoradas

Table 2 - Minimum, maximum and medium values of performance indexes of bull frog tadpoles of the three observed units

\begin{tabular}{|c|c|c|c|c|c|c|c|c|c|}
\hline \multirow[b]{2}{*}{$\begin{array}{l}\text { Ranário } \\
\text { Frog farm } \\
\end{array}$} & \multicolumn{3}{|c|}{$\begin{array}{c}\text { Mortalidade }(\%) \\
\text { Mortality }(\%)\end{array}$} & \multicolumn{3}{|c|}{$\begin{array}{c}\text { Ganho de peso diário }(\mathrm{g}) \\
\text { Daily weight gain }(\mathrm{g})\end{array}$} & \multicolumn{3}{|c|}{$\begin{array}{c}\text { Conversão alimentar (aparente) } \\
\text { Feed: gain ratio (apparent) }\end{array}$} \\
\hline & $\begin{array}{l}\text { Mínimo } \\
\text { Minimum }\end{array}$ & $\begin{array}{l}\text { Máximo } \\
\text { Maximum }\end{array}$ & $\begin{array}{l}\text { Média } \\
\text { Medium }\end{array}$ & $\begin{array}{l}\text { Mínimo } \\
\text { Minimum }\end{array}$ & $\begin{array}{c}\text { Máximo } \\
\text { Maximum }\end{array}$ & $\begin{array}{c}\text { Média } \\
\text { Medium }\end{array}$ & $\begin{array}{l}\text { Mínimo } \\
\text { Minimum }\end{array}$ & $\begin{array}{l}\text { Máximo } \\
\text { Maximum }\end{array}$ & $\begin{array}{c}\text { Média } \\
\text { Medium }\end{array}$ \\
\hline UO-1 & 0 & 26,2 & 2,4 & 0,01 & 0,32 & 0,14 & 0,61 & 2,63 & 1,11 \\
\hline UO-2 & 2,0 & 52,3 & 11,0 & 0,01 & 0,13 & 0,08 & 1,30 & 2,62 & 1,79 \\
\hline UO-3 & 0,0 & 27,5 & 4,3 & 0,06 & 0,22 & 0,12 & 0,84 & 3,00 & 1,59 \\
\hline $\begin{array}{l}\text { Média geral } \\
\text { Average mean }\end{array}$ & 0,7 & 35,3 & 5,9 & 0,03 & 0,22 & 0,11 & 0,92 & 2,75 & 1,50 \\
\hline
\end{tabular}




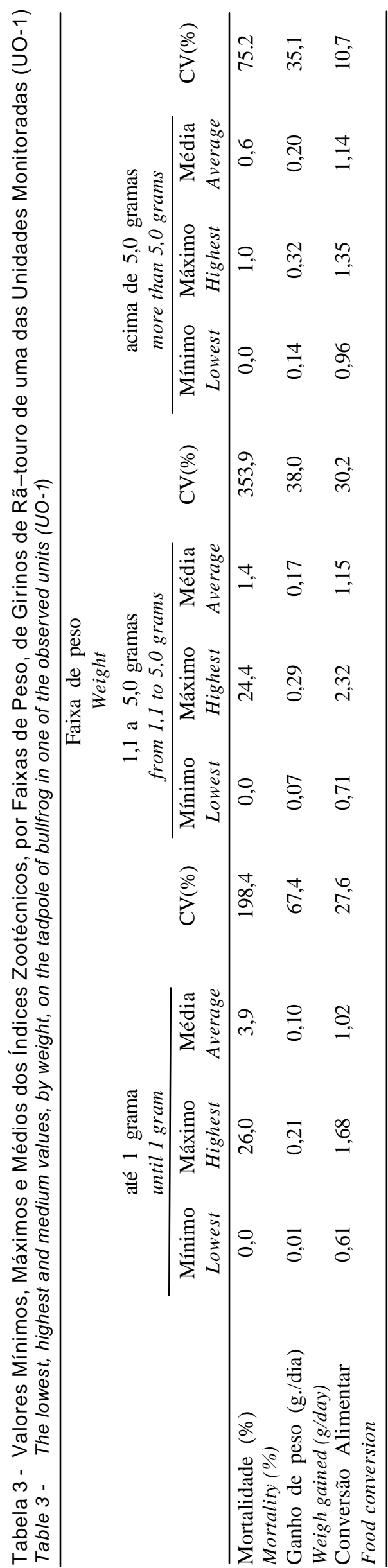

são alimentar (média 1,69:1, para 1,5:1) representa redução nos custos da produção, que se deve não somente à melhoria na qualidade das rações oferecidas atualmente, mas, principalmente, aos benefícios dos ajustes efetuados no manejo de rotina, com a correta oferta do alimento, aliado à padronização da tecnologia (instalações e manejo). Mesma assertiva pode ser denunciada pela redução da mortalidade (7,56 para $5,9 \%$ na média geral), Já o ganho de peso diário seguiu o mesmo padrão anterior, com ligeiro acréscimo $(0,10$ para $0,11 \mathrm{~g} / \mathrm{dia})$.

Com referência aos índices zootécnicos por faixa de peso, nota-se o mesmo desempenho que a maioria das criações tradicionais, onde os níveis são piores durante os primeiros dias de vida (fase inicial), com resultados otimizados após cinco grama, com a mortalidade a $0,6 \%$, ganho de peso 0,20 g/dia, e conversão alimentar de 1,14 : 1, valores que podem ser considerados como satisfatórios e demonstram a eficiência da tecnologia adotada para a criação desses animais.

São apresentados na Tabela 4 os valores de referência, em percentuais, para cálculo do alimento a ser oferecido aos girinos, diariamente, em função do peso médio $(0,1$ a $26 \mathrm{~g})$ e dos intervalos de temperatura (18 a $20^{\circ} \mathrm{C}, 21$ a $23^{\circ} \mathrm{C}, 24$ a $26^{\circ} \mathrm{C}$, e 27 a $29^{\circ} \mathrm{C}$ ), ampliando assim a tabela de alimentação anteriormente proposta por Lima \& Agostinho (1988).

Não foram consideradas as diferenças da qualidade da água disponibilizada para os girinos, ou seja: a exemplo de outros organismos aquáticos cultiváveis, eles também se alimentam de microorganismos e matéria em suspensão, além da ração oferecida, porém em pequena parcela. Em outras palavras, os resultados encontrados em cada ranário se diferenciam, além de outros fatores, pela diferença da qualidade da água disponível. Em dois ranários a origem era de poço semi-artesiano, e um, de uma pequena represa abastecida por fonte natural. Por tais motivos, foram considerados os valores mínimos, máximos e a média geral dos dados obtidos.

A discrepância dos percentuais apresentados na tabela de referência, com os valores encontrados por outros autores (Culley et al., 1977; Fontanello, 1982; Soares et al., 1977), provavelmente seja em razão das grandes diferenças na qualidade das rações empregadas, das características das instalações e das técnicas de manejo utilizadas por estes autores. 
Tabela 4 - Valores de referência para o cálculo de alimentação dos girinos, em função do peso médio $(\mathrm{g})$ e da temperatura da água $\left({ }^{\circ} \mathrm{C}\right)$

Table 4 - Reference values for tadpoles feeding table based on the average weight $(g)$ and water temperature $\left({ }^{\circ} \mathrm{C}\right)$

\begin{tabular}{|c|c|c|c|c|}
\hline $\begin{array}{l}\text { Temperaturas } \\
\text { Temperatures } \\
\mathrm{n}^{\circ} \text { de parcelas } \\
n^{\circ} \text { portions }\end{array}$ & 18 a $20^{\circ} \mathrm{C}$ & $\begin{array}{c}21 \text { a } 23^{\circ} \mathrm{C} \\
5\end{array}$ & $\begin{array}{c}24 \text { a } 26^{\circ} \mathrm{C} \\
6\end{array}$ & $\begin{array}{c}27 \text { a } 29^{\circ} \mathrm{C} \\
7\end{array}$ \\
\hline $\begin{array}{l}\text { Peso médio dos girinos } \\
\text { Tadpoles average weight }\end{array}$ & $\begin{array}{c}\text { Consumo } \\
\text { estimado }(\%) \\
\text { Estimated } \\
\text { intake }\end{array}$ & $\begin{array}{c}\text { Consumo } \\
\text { estimado (\%) } \\
\text { Estimated } \\
\text { intake }\end{array}$ & $\begin{array}{c}\text { Consumo } \\
\text { estimado (\%) } \\
\text { Estimated } \\
\text { intake }\end{array}$ & $\begin{array}{c}\text { Consumo } \\
\text { estimado (\%) } \\
\text { Estimated } \\
\text { intake } \\
\end{array}$ \\
\hline 0,1 & 8,00 & 9,0 & 11,7 & 13,6 \\
\hline 0,2 & 7,00 & 7,5 & 9,0 & 10,4 \\
\hline 0,3 & 6,80 & 6,8 & 8,2 & 9,5 \\
\hline 0,4 & 6,50 & 6,5 & 7,8 & 9,0 \\
\hline 0,5 & 6,00 & 6,3 & 7,6 & 8,8 \\
\hline 0,6 & 5,90 & 6,3 & 7,6 & 8,8 \\
\hline 0,7 & 5,80 & 6,3 & 7,5 & 8,7 \\
\hline 0,8 & 5,70 & 6,2 & 7,5 & 8,6 \\
\hline 0,9 & 5,60 & 6,2 & 7,4 & 8,6 \\
\hline 1 & 5,50 & 6,1 & 7,3 & 8,4 \\
\hline 2 & 4,40 & 5,2 & 6,2 & 7,2 \\
\hline 3 & 4,00 & 4,8 & 5,6 & 6,5 \\
\hline 4 & 3,80 & 4,6 & 5,5 & 6,3 \\
\hline 5 & 3,40 & 4,3 & 5,3 & 6,2 \\
\hline 6 & 3,20 & 4,0 & 5,0 & 5,8 \\
\hline 7 & 2,80 & 3,5 & 4,4 & 5,1 \\
\hline 8 & 2,60 & 3,3 & 4,1 & 4,7 \\
\hline 9 & 2,30 & 2,9 & 3,6 & 4,2 \\
\hline 10 & 2,10 & 2,6 & 3,3 & 3,6 \\
\hline 11 & 1,90 & 2,4 & 3,0 & 3,3 \\
\hline 12 & 1,70 & 2,1 & 2,7 & 2,8 \\
\hline 13 & 1,55 & 1,9 & 2,4 & 2,4 \\
\hline 14 & 1,45 & 1,8 & 2,3 & 2,3 \\
\hline 15 & 1,35 & 1,7 & 2,1 & 2,1 \\
\hline 16 & 1,25 & 1,6 & 2,0 & 2,0 \\
\hline 17 & 1,20 & 1,5 & 1,9 & 1,9 \\
\hline 18 & 1,10 & 1,4 & 1,4 & 1,4 \\
\hline 19 & 1,05 & 1,3 & 1,3 & 1,3 \\
\hline 20 & 1,00 & 1,3 & 1,3 & 1,3 \\
\hline 21 & 0,95 & 1,2 & 1,2 & 1,2 \\
\hline 22 & 0,90 & 1,1 & 1,1 & 1,1 \\
\hline 23 & 0,85 & 1,1 & 1,1 & 1,1 \\
\hline 24 & 0,80 & 1,0 & 1,0 & 1,0 \\
\hline 25 & 0,75 & 0,9 & 0,9 & 0,9 \\
\hline 26 & 0,70 & 0,88 & 0,88 & 0,88 \\
\hline
\end{tabular}

\section{Conclusões}

A tabela de alimentação para girinos de rã-touro aqui proposta, apresenta valores gradativos para se efetuar o arraçoamento quando a temperatura do tanque apresentar intervalos de temperatura de 18 a $20^{\circ} \mathrm{C}, 21$ a $23^{\circ} \mathrm{C} ; 24$ a $26^{\circ} \mathrm{C}$; e 27 a $29^{\circ} \mathrm{C}$. Ela foi aferida no decorrer do monitoramento através dos indicadores zootécnicos estimados, demonstrando sua eficiência, como valores de referência para o manejo alimentar de rotina. Tais valores podem, e devem, sofrer alteração, dependendo da qualidade da ração a ser oferecida e água disponível. Portanto esses valores devem ser novamente calibrados toda vez que houver o emprego de rações de qualidade nutricional distintas da empregada nesta pesquisa (ração para truta, extrusada, fase inicial).

Os resultados do desempenho dos animais corroboraram para a constatação da adequação das instalações e técnicas de manejo propostas para o Sistema Anfigranja de criação intensiva de rãs. 


\section{Literatura Citada}

BRATTSTROM, B.H. Amphibian temperature regulation studies in the field and laboratory.America Zoological, v.19 (único), p. 345-356, 1979.

CULLEY, D.; MEYERS, S.S.P.; DOUCETTE JR., A.J. A high density rearing system for larval anurans. Laboratory Animal v.6, p.34-41, 1977.

FONTANELLO, D.; SOARES, H.A.; MANDELLI JR., J. Crescimento de girinos de Rana catesbeiana criados com diferentes quantidades de ração Boletim Instituto de Pesca, v.9 (único), p.39-44, 1982.

LIMA, S.L. A criação de rãs (Sistema Anfigranja), manual técnico CPT, Viçosa, nº. 08, 1997, 48p.

LIMA, S.L.; VALLES, G. A. Desenvolvimento de instalações e Técnicas de Manejo para a criação intensiva de girinos de rãtouro, Rana catesbeiana, no sistema anfigranja. In: SIMPÓSIO BRASILEIRO DE AQÜICULTURA, 10., 1998, Recife. Resumos... Recife: Associação Brasileira de Aqüicultura, 1998. p. 273.

LIMA, S.L.; AGOSTINHO, C.A. A criação de rãs. Rio de Janeiro: Editora Globo, 1988. 172p.

LIMA, S.L.; AGOSTINHO, C.A. A tecnologia de criação de rãs. Viçosa, MG: Universidade Federal de Viçosa, 1992. 168p.
LIMA, S.L.; LANZA, A.L.; CASALI, A.P. et al. Sistema anfigranja: índices de produtividade com rã-touroRana catesbeiana. In: TECHNOFROG' 97, 1997, Santos.Anais... Santos: Academia de Estudos Técnicos em Ranicultura, 1997. p.198-199.

LIMA, S.L; CRUZ, T.A.; MOURA, M.O. Ranicultura: análise da cadeia produtiva. Viçosa, MG: Editora Folha de Viçosa, 1999. 170p.

MELO S.C.P.; SEIXAS FILHO, J.T.; VEIGA, R.C.A. et al. Avaliação do desempenho de girinos de Rana catesbeiana, alimentados com diferentes proporções de ração em relação ao peso corporal. In: ENAR \& TECHNOFROG'97, 9., 1997, Santos. Anais... Santos: Academia Brasileira de Estudos Técnicos em Ranicultura, 1997. p.196.

SOARES, H.A.; MANDELLI JR., J.; JUSTO, C.L. et al. Desenvolvimento Ponderal de girinos de Rana catesbeiana, em criação intensiva, com diferentes quantidades de alimento. Boletim Instituto de Pesca v.12, n.2, p.55-59, 1985.

Recebido em: $18 / 12 / 01$

Aceito em: 13/11/02 